\title{
ILUSTRAÇÃO DE CARACTERES MICROSCÓPICOS DE DROGAS VEGETAIS PARA O CONTROLE DE QUALIDADE FARMACOGNÓSTICO. III. ERVA-MATE (Ilex paraguariensis A. ST.-HIL., AQUIFOLIACEAE)
}

\section{ILLUSTRATION OF VEGETAL DRUG MICROSCOPIC CHARACTERS FOR THE PHARMACOGNOSTIC QUALITY CONTROL. III. YERBA MATÉ (Ilex paraguariensis A. ST.-HIL., AQUIFOLIACEAE)}

\author{
DUARTE, M. do R.; GOLAMBIUK, G. \\ Laboratório de Farmacognosia, Departamento de Farmácia, Universidade Federal do Paraná \\ (UFPR), Av. Pref. Lothário Meissner, 632, Jardim Botânico, 80210-170, Curitiba, PR, \\ marciard@ufpr.br \\ RECEBIDO: 10/01/08 ACEITO:03/02/08
}

\begin{abstract}
RESUMO:
Em continuidade aos artigos anteriores de ilustração de caracteres microscópicos, considerados úteis como recurso complementar para a identificação farmacopéica de drogas vegetais, este trabalho objetivou ilustrar as estruturas anatômicas da erva-mate (Ilex paraguariensis A. St.Hil., Aquifoliaceae). Essa espécie é considerada medicinal, constando de monografias farmacopéicas, e o seu consumo na forma de bebida é significativo para a indústria de alimentos. O material vegetal foi coletado no Jardim Botânico Municipal de Curitiba e identificado por taxonomista. Para a elaboração de lâminas semipermanentes, amostras foliares foram fixadas, seccionadas nos sentidos transversal e longitudinal, e coradas. Dentre as estruturas representadas, destaca-se que a folha é hipoestomática, havendo predominância de estômatos anomocíticos inseridos no mesmo nível das células circunvizinhas. O mesofilo é dorsiventral e a nervura central biconvexa possui um feixe vascular colateral em disposição cêntrica. Drusas e prismas de oxalato de cálcio estão presentes.
\end{abstract}

Palavras-chave: Droga vegetal, farmacopéia, ilustração, microscopia.

\begin{abstract}
:
Similarly to the previous papers that dealt with the illustration of anatomical characters, useful as an additional resource for the identification of pharmacopoeical vegetal drugs, this work has aimed to illustrate the anatomical structures of yerba maté (Ilex paraguariensis A. St.-Hil., Aquifoliaceae). This species is considered medicinal, being described in pharmacopoeical monographies, and relevant for the food industry. The plant material was collected at the Jardim Botânico Municipal de Curitiba and identified by a taxonomist. For semi-permanent slides, leaf samples were fixed, sectioned transversal and longitudinally, and stained. Among the structures depicted, this study shows hypostomatic leaf, having predominantly anomocytic stomata on the same level as the surrounding cells. The mesophyll is dorsiventral and the midrib is biconvex, showing one collateral vascular bundle in centric arrangement. Druses and prisms of calcium oxalate are present.
\end{abstract}

Keywords: Illustration, microscopy, pharmacopoeia, vegetal drug.

\section{INTRODUÇÃO}

Na realização de análises farmacognósticas, a confirmação da identidade das drogas 
vegetais é feita com base nas descrições macroscópicas e microscópicas, as quais tratam respectivamente dos caracteres morfológicos externos e anatômicos a serem observados. 0 reconhecimento das estruturas é facilitado quando as descrições são acompanhadas de ilustrações.

Artigos publicados anteriormente neste periódico objetivaram apresentar ilustrações que complementassem as descrições morfológicas de drogas vegetais farmacopéicas. Em continuidade a essas análises, este trabalho apresenta as ilustrações correspondentes à ervamate, cuja monografia consta da Farmacopéia Brasileira I (SILVA, 1926) e da Farmacopéia Britânica (BHP, 1983; 1996).

A erva-mate consiste de folhas obtidas da espécie llex paraguariensis A. St.-Hil., Aquifoliaceae (SILVA, 1926; BHP, 1983; 1996), nativa da Argentina, do Paraguai e do sul do Brasil (BHP, 1996; FETROW; AVILA, 2000). É uma árvore de $20 \mathrm{~m}$ de altura, copa densa (Fig. 1), folhas simples, alternas, oblongas ou obovadas, curto-pecioladas, coriáceas, com margem crenada ou serreada e 6 a 20 cm de comprimento (Fig. 2) (BHP, 1996; LORENZI; MATOS, 2002).

Popularmente, é conhecida como mate, chá-do-paraguai e congonha em português (NEWALL et al., 2002), yerba del Paraguaye té de los Jesuitas em espanhol (ALONSO, 1998) e como yerba maté e Paraguay tea em inglês (SKIDMORE-ROTH, 2004).

De acordo com Newall et al. (2002), as folhas apresentam alcalóides xantínicos, na proporção de 0,2 a $2 \%$ de cafeína [1], 0,1 a 0,2\% de teobromina e $0,05 \%$ de teofilina. Foram também isolados os flavonóides campferol [2], quercetina e seus glicosídeos, como também os terpenóides ácido ursólico [3] e b-amirina. Segundo padrões farmacopéicos, a droga deve conter no mínimo 1,5\% de cafeína (SILVA, 1926), até 16\% de taninos (BHP, 1983) e não exceder $2 \%$ de matéria orgânica estranha (BHP, 1996).<smiles>Cn1c(=O)c2c(ncn2C)n(C)c1=O</smiles>

1<smiles>O=c1cc(-c2ccc(O)c(O)c2)oc2cc(O)cc(O)c12</smiles>

2<smiles>CC1CCC2(C(=O)O)CCC3(C)C(=CCC4C5(C)CCC(O)C(C)(C)C5CCC43C)C2C1C</smiles>

3

O uso fitoterápico da erva-mate pode ser atribuído às ações farmacológicas da cafeína, que se traduzem por estimulação do sistema nervoso central e efeitos diurético, lipolítico e glicogenolítico (ALONSO, 1998; SKIDMORE-ROTH, 2004).

Para o preparo da droga, ramos folhados do vegetal são aquecidos rapidamente no fogo (sapecados), de modo a favorecer a inativação enzimática. Em seguida, as folhas são destacadas do caule, dessecadas pelo calor e rasuradas (cancheadas). Esse material é ensacado e armazenado por um período aproximado de um ano, propiciando a formação de compostos aromáticos característicos (EVANS et al., 2002). O produto final é geralmente consumido na forma de bebida gelada (tererê) ou de 
infusão (chimarrão). Adicionalmente, as folhas podem sofrer um processo de torrefação e gerar a matéria-prima para o chá mate tostado.

\section{MATERIAL E MÉTODOS}

O material botânico foi coletado de exemplares do Arboreto do Jardim Botânico Municipal de Curitiba, localizado na latitude de $25^{\circ} 26^{\prime} \mathrm{S}$, longitude de $49^{\circ} 14^{\prime} \mathrm{W}$ e altitude de $930 \mathrm{~m}$, em novembro de 2007. A identificação taxonômica correspondeu à exsicata registrada como MBM 318474.

Amostras de folhas adultas foram fixadas em FAA 70 (JOHANSEN, 1940) e posteriormente armazenadas em etanol a 70\% (BERLYN; MIKSCHE, 1976). Fragmentos foliares foram seccionados à mão livre, nos sentidos paradérmico, transversal e longitudinal, e corados com azul de astra e fucsina básica (ROESER, 1972). As estruturas anatômicas foram fotografadas no microscópio de luz Olympus BX40.

\section{RESULTADOS E DISCUSSÃO}

A lâmina foliar, em vista frontal, mostra células epidérmicas praticamente com contorno poligonal e de paredes anticlinais espessadas (Fig. 3, 4). Na superfície adaxial, as células epidérmicas são comparativamente maiores (Fig. 3, 4). Ocorrem numerosos estômatos exclusivamente na face abaxial, havendo predominância do tipo anomocítico (Fig. 4). Estes são ladeados por quatro a seis células e estão inseridos no mesmo nível das demais células circunvizinhas. O sistema de revestimento é recoberto por uma cutícula relativamente espessada (Fig. 7) e estriada (Fig. 5, 7).

Em secção transversal, o mesofilo é dorsiventral (Fig. 6), compreendendo cerca de dois estratos de parênquima paliçádico (Fig. 7) e de sete camadas de parênquima esponjoso, cujas células deixam consideráveis espaços intercelulares (Fig. 7).

A nervura central é biconvexa, exibindo uma curvatura proeminente na superfície abaxial (Fig. 6). Raros tricomas tectores unicelulares, retos e de paredes espessadas, podem ser encontrados. Subjacente à epiderme, ocorrem algumas camadas de colênquima angular junto a ambas as faces. Observa-se um feixe vascular colateral, de disposição cêntrica (Fig. 6, 9) e circundado por uma bainha esclerenquimática (Fig. 6, 8). Idioblastos contendo prismas e, freqüentemente, drusas de oxalato de cálcio estão presentes (Fig. 8, 9).

O pecíolo (Fig. 10), seccionado transversalmente, é plano a levemente côncavo na superfície adaxial e nitidamente convexo na face oposta. No nível mediano, é formado de um feixe vascular colateral, em disposição cêntrica, e de dois feixes acessórios junto à face adaxial. As demais características são semelhantes às da nervura central.

De um modo geral, os caracteres anatômicos ilustrados neste trabalho correspondem às descrições microscópicas das monografias da erva-mate, incluídas na Farmacopéia Brasileira I (SILVA, 1926) e na Farmacopéia Britânica (BHP, 1983; 1996). No entanto, a menção de células epidérmicas de paredes delgadas na face abaxial e de estômatos com célulasguarda lignificadas (BHP, 1983) não foi confirmada neste estudo. Diferentemente do formato reniforme do feixe vascular da nervura central, descrito na Farmacopéia Brasileira I (SILVA, 1926), nesta investigação o mesmo se apresentou com arranjo cêntrico. 

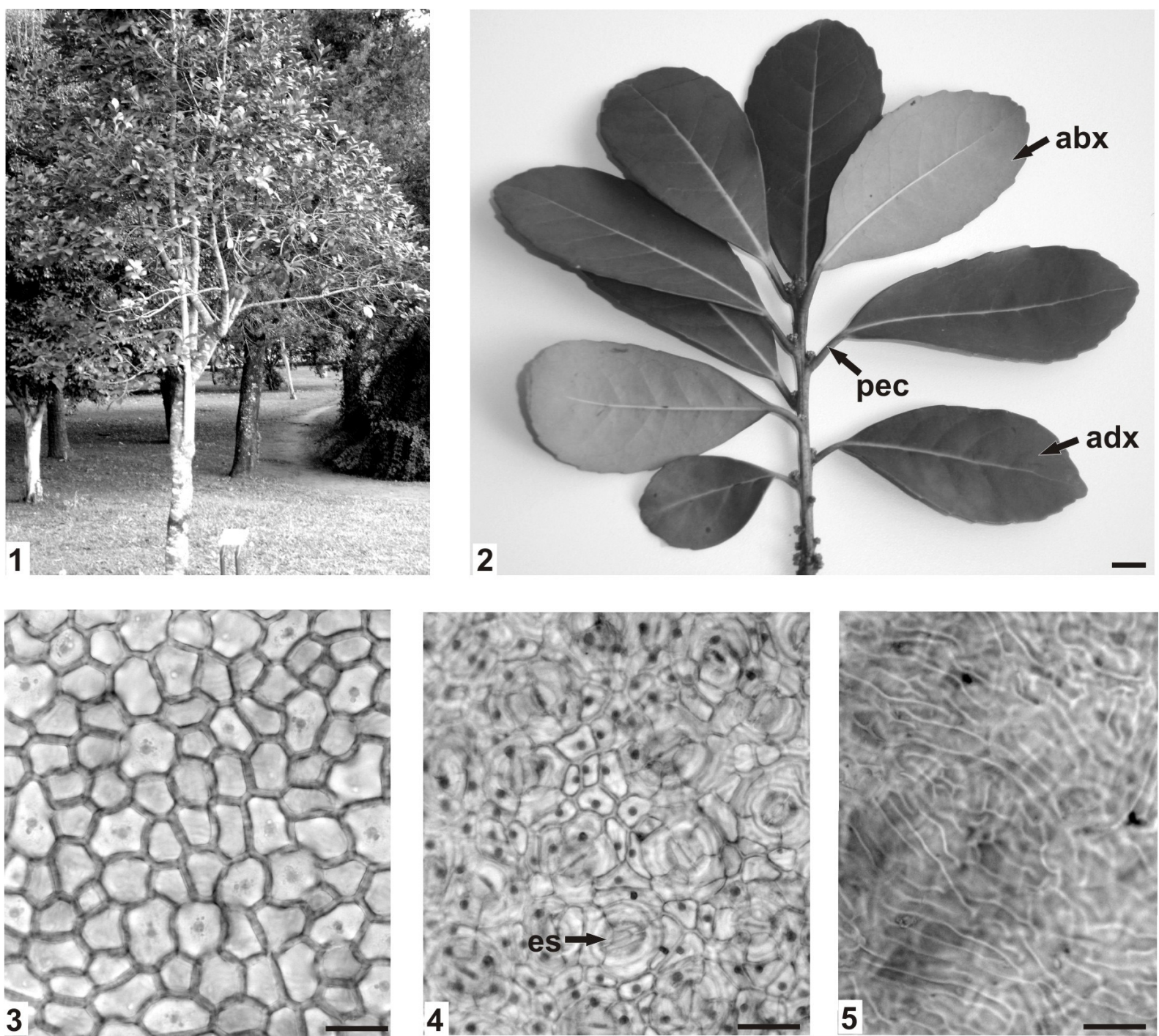

FIGURAS 1-5. ERVA-MATE (Ilex paraguariensis A. ST.-HIL., AQUIFOLIACEAE): 1. ASPECTO GERAL DA PLANTA NO HÁBITO; 2. RAMO FOLIAR APICAL; 3, 4. VISTA FRONTAL DA EPIDERME FOLIAR, FACES ADAXIAL E ABAXIAL, RESPECTIVAMENTE; 5. DETALHE DA CUTÍCULA ESTRIADA QUE REVESTE A EPIDERME FOLIAR. ABX - FACE ABAXIAL DA EPIDERME, ADX - FACE ADAXIAL DA EPIDERME, ES - ESTÔMATO, PEC - PECÍOLO. $\operatorname{BARRA}=1,5 \mathrm{~cm} \mathrm{(2),} 20 \mathrm{~mm}(3-5)$

Com relação às figuras de Jackson e Snowdon (1990), elaboradas com a erva-mate em pó, há semelhança nas representações microscópicas feitas por esses autores e as apresentadas neste estudo, como seria de se esperar, o que confirma a coerência entre as descrições e as ilustrações que as representam. 

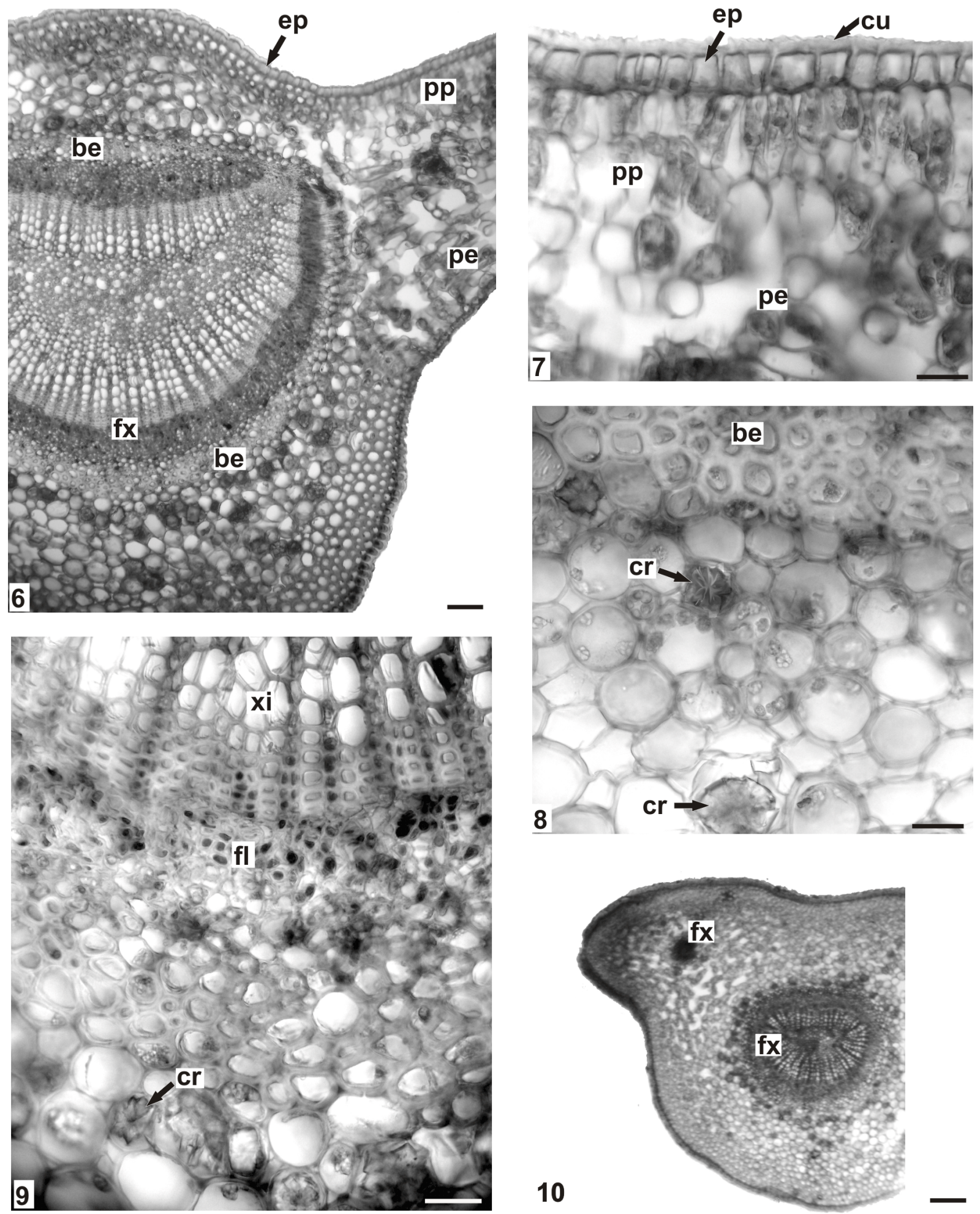

FIGURAS 6-10. ERVA-MATE (Ilex paraguariensis A. ST.-HIL., AQUIFOLIACEAE) - SECÇÃO TRANSVERSAL DA FOLHA: 6. NERVURA CENTRAL E PARTE DA REGIÃO INTERNERVURAL, MOSTRANDO MESOFILO DORSIVENTRAL E FEIXE VASCULAR COLATERAL; 7. PORÇÃO DO LIMBO FOLIAR, EM QUE SE NOTAM EPIDERME UNISSERIADA E CLORÊNQUIMA; 8. PARTE DA BAINHA ESCLERENQUIMÁTICA E CRISTAIS DE OXALATO DE CÁLCIO NA NERVURA CENTRAL; 9. DETALHE DO FEIXE VASCULAR COLATERAL; 10. PECÍOLO. BE - BAINHA ESCLERENQUIMÁTICA, CR - CRISTAL DE OXALATO DE CÁLCIO, CU - CUTÍCULA, EP EPIDERME, FL - FLOEMA, FX -FEIXE VASCULAR, PE - PARÊNQUIMA ESPONJOSO, PP - PARÊNQUIMA PALIÇÁDICO, XI - XILEMA. BARRA = $100 \mathrm{~mm}(10), 50 \mathrm{~mm}$ (6), $20 \mathrm{~mm}$ (7-9) 


\section{REFERÊNCIAS}

ALONSO, J. R. Tratado de fitomedicina: bases clínicas y farmacológicas. Buenos Aires: Isis, 1998.

BERLYN, G. P.; MIKSCHE, J. P. Botanical microtechnique and cytochemistry. Ames: lowa State University Press, 1976.

BHP: British Herbal Pharmacopoeia. 2nd ed. Bournemouth: British Herbal Medicine, 1983.

BHP: British Herbal Pharmacopoeia. 4th ed. Exeter: British Herbal Medicine, 1996.

EVANS, W. C. Trease and Evans pharmacognosy. 15th ed. Edinburgh: W.C. Saunders, 2002.

FETROW, C. W.; AVILA, J. R. Manual de medicina alternativa. Rio de Janeiro: Guanabara Koogan, 2000.

JACKSON, B. P.; SNOWDON, D. W. Atlas of microscopy of medicinal plants, culinary herbs and spices. London: Belhaven, 1990.

JOHANSEN, D. A. Plant microtechnique. New York: McGraw-Hill Book, 1940.

LORENZI, H.; MATOS, F. J. A. Plantas medicinais do Brasil: nativas e exóticas. Nova Odessa: Plantarum, 2002.

NEWALL, C. A.; ANDERSON, L. A.; PHILLIPSON, J. D. Plantas medicinais. São Paulo: Premier, 2002.

ROESER, K. R. Die Nadel der Schwarzkiefer-Massenprodukt und Kunstwerk der Natur. Mikrokosmos, Stuttgart, v. 61, p. 33-6, 1972.

SILVA, R. A. D. Pharmacopéia dos Estados Unidos do Brasil. São Paulo: Nacional, 1926.

SKIDMORE-ROTH, L. Handbook of herbs and natural supplements. 2nd ed. St. Louis: Mosby, 2004. 